\title{
Monomorphs, Bimorphs, and Multimorphs from Polar Polymer Electrets
}

\author{
Simona Bauer-Gogonea, Siegfried Bauer \\ Angewandte Physik \\ Johannes-Kepler-Universität Linz \\ Altenbergerstr. 69, A-4040 Linz, Austria \\ and \\ Reimund Gerhard-Multhaupt \\ Angewandte Physik kondensierter Materie \\ Institut für Physik, Universität Potsdam \\ Am Neuen Palais 10, D-14469 Potsdam, Germany \\ Received 22 January, 1998
}

\begin{abstract}
Mono-, bi-, and multimorphs are poled electrets with $+/ 0,+/-$, and more complicated alternatingpolarity dipole-orientation patterns, respectively. Because of their unusual macroscopic piezo- and pyroelectrical as well as nonlinear optical properties, such structures are of particular interest for special transducer and optical waveguide applications. In this contribution, the preparation of such dipole-polarization patterns in ferroelectric and amorphous polymer electrets and their nondestructive probing by means of thermal and acoustical techniques are reviewed.
\end{abstract}

\section{Introduction}

Already in their early studies of piezoelectricity, Jacques and Pierre Curie, who had discovered the effect in 1880, employed three-layer samples both for the direct and the converse piezoelectric effect [1]. With two inner electrodes between the three individual layers, the $+/-/+$ arrangement served mainly two purposes: To increase the sensitivity and to provide shielding of the inner electrodes, while the outer electrodes were always grounded [1]. Today, similar arrangements are still used in order to enhance the sensitivity of piezoelectric-polymer transducers [2], but in addition, multilayer structures without inner electrodes are now used as code-specific transmitters and detectors [2] or as monomorphs, bimorphs and multimorphs (see below).

Monomorphs, bimorphs, and multimorphs are poled electrets with $+/ 0,+/-$, and more complicated alternating-polarity dipole-orientation patterns, respectively, across their thickness. They often possess unusual macroscopic piezoelectrical, pyroelectrical, or nonlinear optical properties which make them particularly useful for special transducer or waveguide applications (see below). However, the preparation of poled polymer electrets with well-defined profiles of their internal dipole polarization is not possible without suitable probing techniques.

In one of the very early studies on the probing of internal charge and polarization profiles, beeswax electret samples were sliced with a chrome-nickel blade, and the charge on each slice was determined by means of a Faraday cage and an electroscope [3]. This and several later sectioning experiments on electrets are discussed in the still very useful bibliographical review of Gross [4]. It was, however, also shown by Gross and Moraes [5] that the profile of a true volume polarization can only be reliably obtained by electroding the slices in order to eliminate the surface charge generated in the cutting process and by then determining the total depolarization current of each slice. With this improved sectioning technique, it could be demonstrated that carnauba wax electrets do indeed possess a true volume polarization (heterocharge) in addition to the injected and trapped space charge (homocharge) [5].

In the mid-1970s, Peterson et al. [6,7] and Collins [8-10] independently showed that thermal waves or pulses may be employed for the nondestructive probing of charge and polarization profiles in electrets. 
Later, Lewiner et al. [11-13] and Rozno and Gromov $[14,15]$ demonstrated the use of high-frequency acoustical waves for the same purpose. Today, a whole range of thermal and acoustical probing techniques for electrets is available. Here, only those which have been utilized for the control of polarization profiles in polar polymers are of interest, i.e. the thermal-pulse or heat-pulse technique $[7,9,16]$ and the thermal-wave or laser-intensity modulation method $[6,17,18]$ on the one hand and the laser-induced pressure-pulse or pressurewave propagation method $[13,19,20]$ and the piezoelectrically generated pressure-step technique $[19,21]$ on the other hand.

Among the first studies into monomorphs, bimorphs, and multimorphs from poled polymers were investigations of sandwiches consisting of two or more separate films [7,22-24]. These film sandwiches were either poled as a whole and the films measured individually $[7,22,23]$, or the films were poled separately and measured in a sandwich [24] which is the same method as that of the Curie brothers [1].

In contrast to this approach, we discuss here mainly monolithic samples in which the desired polarization profiles are produced by means of selective poling techniques, sometimes in combination with specifically chosen non-uniform sample properties. Charge injection with a mono-energetic electron beam or from metal electrodes may be used to produce monomorph or bimorph polarization profiles; these approaches are briefly described in the following sections II and III. Two-step poling of double or multiple layers with alternating high and low Curie or glass-transition temperatures is discussed in sections IV and V. Selective photo-induced poling of nonlinear optical (NLO) polymers is reviewed in section VI, while a selected range of suggested and demonstrated applications are mentioned in the final section VII.

\section{Electron-beam poling}

The study of the charge dynamics [25] and of the resulting space-charge profiles $[15,26]$ in mostly nonpolar polymer electrets during and after electron-beam irradiation (see also the contribution by Sessler and Yang in this special issue) led to the observation that well-confined space-charge layers are usually generated if the primary electrons all have approximately the same energy. Using this thin charge layer as a "virtual electrode" inside the polymer film, Gross et al. first demonstrated the selective poling of piezo- and pyroelectric polyvinylidenefluoride (PVDF) electrets across their thickness by means of mono-energetic electron beams [27]. The principle of this poling method is schematically shown in the right half of Fig. 4 below. Several non-uniform piezoelectricity profiles were generated with primary electron-beam energies of 5, 10, 15, 20 , and $28 \mathrm{keV}$ and probed with laser-induced pressure pulses [27]. The thickness of the unpoled (i.e. passive) volume in the resulting monomorphs is a function of the electron range.

An independent measure of the remanent polarization achieved during electron-beam poling is obtained by discharging the sample surface with a liquid contact and charging it again during a second $12 \mathrm{keV}$ electronbeam irradiation. According to Fig. 1, a polarization of approximately $0.12 \mathrm{C} / \mathrm{m}^{2}$ is found from the integral over the difference between the first poling current (I), which includes the orientation of the remanent polarization between deposited charge layer and rear electrode, and the second current curve (II), which represents only the charge replacement together with a re-orientation of the non-permanent dipole polarization [27].

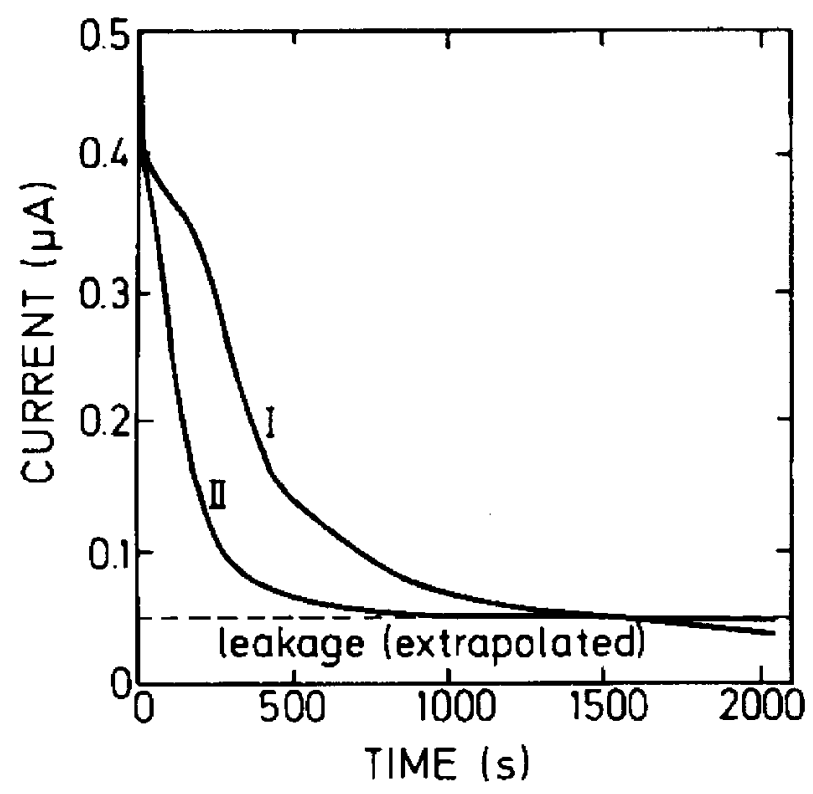

Figure 1: Poling currents during electron-beam irradiation of a 32- $\mu \mathrm{m}$ polyvinylidenefluoride film: Virgin sample (I) and poled sample after discharge of its free surface (II). Because of unavoidable differences in the electron-beam current, curve II was adjusted with an affine coordinate transformation (after [27]).

In a related study, Fukada et al. poled PVDF films from two different manufacturers with a $30 \mathrm{keV}$ electron 
beam, probed the resulting monomorphs with laserinduced pressure pulses, and observed the expected bending piezoelectricity of the monomorphs [28]. This study also contains a theoretical discussion of the piezoelectric behavior to be expected from monomorphs and bimorphs with symmetric and asymmetric polarization profiles [28]. Subsequent investigations revealed that various piezoelectricity profiles may be produced by means of high-field, thermal, corona, and electron-beam poling as schematically shown in Fig. 2 [29]. Application of electron-beam poling from both sides led to a rather symmetric bimorph instead of the monomorph obtained with one-sided electron-beam poling [29].
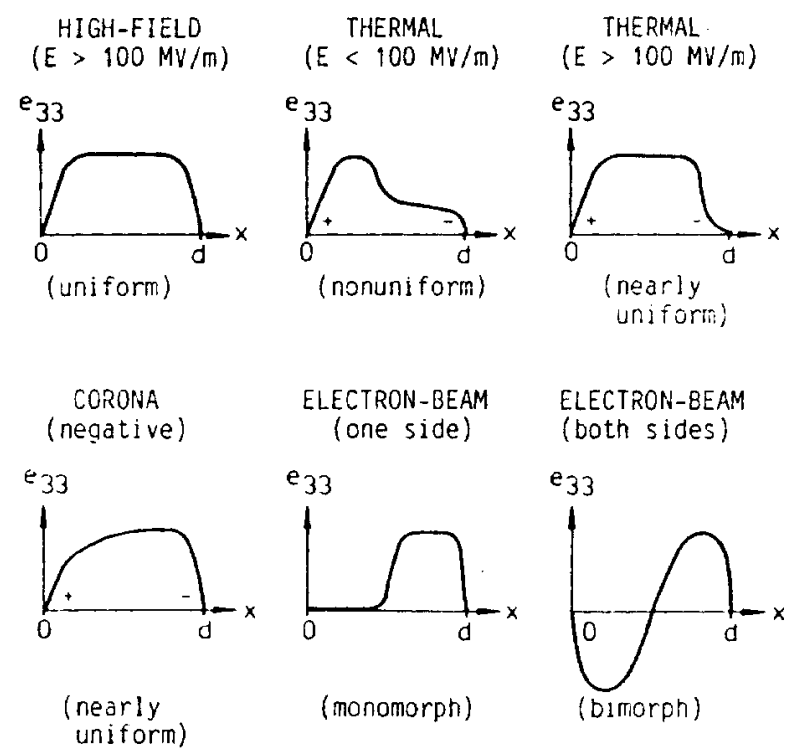

(monomorph)

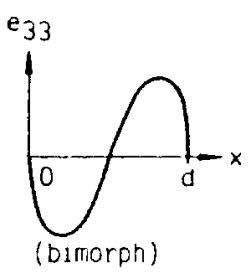

Figure 2: Schematic piezoelectricity (or dipole-polarization) profiles in ferroelectric polymer electrets of thickness d after poling with various techniques as indicated (after [29]).

Closer examination of tensile and bending piezoelectricity in PVDF monomorphs and bimorphs showed that the respective coefficients can be optimized by a proper choice of poling parameters and that the tensile piezoelectricity decays at lower temperatures than the bending piezoelectricity, at least under the conditions used in that study [30]. The length dependence of the three lowest resonance frequencies and of the considerable deflection under DC bias of an electron-beam poled PVDF monomorph is depicted in Fig. 3 [30].

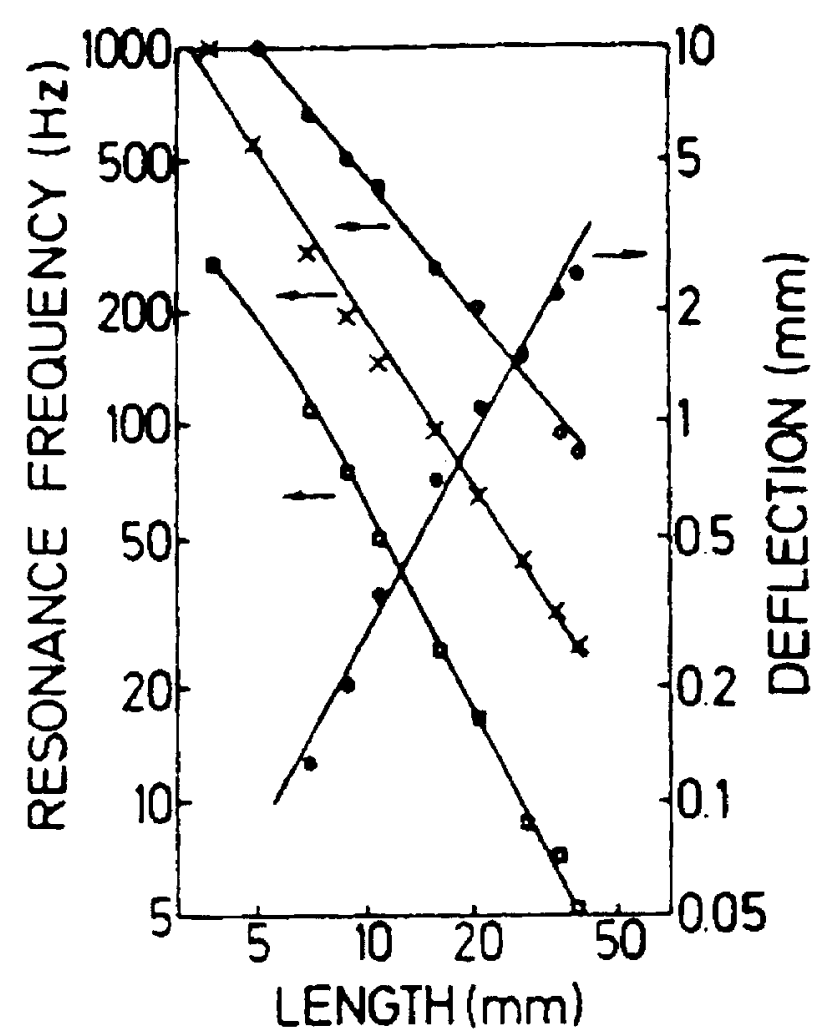

Figure 3: Influence of the free length on the three lowest resonance frequencies (left-side ordinate) and on the deflection (right-side ordinate) of a PVDF monomorph (after [30]).

Instead of the defocused large-area electron-beam irradiation of the poling experiments discussed so far, Schilling et al. employed a narrowly focussed electron beam and scanned it across a relatively small sample area [31]. Primary electron-beam energies of 5, 10, 20, and $30 \mathrm{keV}$ were utilized, and the deposited charge and thus also the electric field were varied by changing the area scanned during a fixed period of time [31]. The resulting non-uniform piezoelectricity profiles were probed with piezoelectrically generated pressure steps [21,31]. Among other results, it was found (a) that a bimorph could be produced by reversing part of the polarization in a previously uniformly poled PVDF film, (b) that the magnitude of the polarization increased with increasing electron-beam energy (probably because the effective poling field is higher for smaller thicknesses of the poled volume), and (c) that there is no sharp boundary between unpoled and poled volume with this poling method (probably because a well-confined charge layer forms only with a defocussed electron beam) [31].

In order to optimize the overlap integral between the fundamental optical wave, its second harmonic, and the active volume of the nonlinear optical (NLO) polymer in a frequency- doubling waveguide, electron-beam 
poling of the NLO polymer was suggested and demonstrated [32]. The concepts of the poling method and of the 0/1-mode frequency conversion are schematically illustrated in Fig. 4 where the waveguide must of course be operated with a lower cladding above the bottom electrode [32]. Laser-induced pressure pulses as well as frequency-scanned thermal waves were employed to probe the achieved dipole-orientation profile. In addition, electro-optical and thermally stimulated depolarization measurements were made in order to independently confirm the NLO activity and the dipole orientation in the polymer film [32].

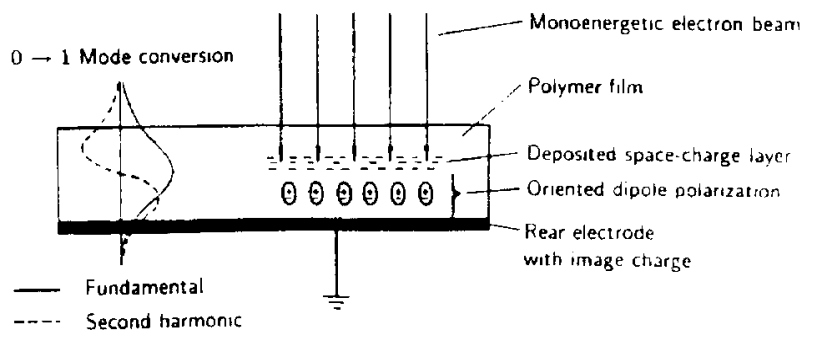

Figure 4: Concept of 0/1-mode second-harmonic generation in a nonlinear optical waveguide (left) and of its selective poling by means of electron beam (right) (after [32]).

\section{Non-uniform poling with charge injection}

With all poling methods for piezo- and pyroelectric or nonlinear optical polymer electrets, non-uniform polarization profiles are found under certain conditions such as overall fields below the coercive field or high conductivities caused e.g. by increased temperatures or by impurities. Examples of non-uniform polarization profiles resulting from charge transport are given in the literature e.g. for corona poling [19], for electron-beam poling [31], or for electrode poling [33]. Prior to the wide-spread use of nondestructive high-resolution probing techniques, the poling of only part of the sample volume led sometimes to erroneous conclusions about the coercive field and its temperature dependence in ferroelectric polymers because it was not yet possible to probe the internal distribution of the dipole polarization.

By use of piezoelectrically generated pressure steps for probing the piezoelectricity profiles during and after poling, Eisenmenger et al. demonstrated in several studies [33- 37] (see also their contribution in this special issue) that (1) charge injection from the electrodes and charge trapping at crystalline-amorphous interfaces play an essential role in the orientation and stabilization of the ferroelectric polarization in PVDF and its copolymers with trifluoroethylene (TrFE) and (2) that the underlying mechanisms may be employed for the generation of monomorphs, bimorphs, and multimorphs. As examples, so-called trimorphs with $+/ 0 /+$ and $+/-/+$ profiles are shown together with snapshots from their development in Figs. 5 and 6 , respectively. The $+/ 0 /+$ trimorph of Fig. 5 was formed by first uniformly poling a $38 \mu \mathrm{m}$ thick PVDF film under a field of $180 \mathrm{MV} / \mathrm{m}$ and then depolarizing its central part under a field of only $60 \mathrm{MV} / \mathrm{m}$, but with opposite polarity [35]. The $+/-/+$ trimorph of Fig. 6 was generated with 80 poling pulses of alternating polarity at a field amplitude of 120 $\mathrm{MV} / \mathrm{m}$, a pulse duration of $0.1 \mathrm{~s}$, and a pulse-repetition rate of $1 \mathrm{~s}^{-1}[33]$.
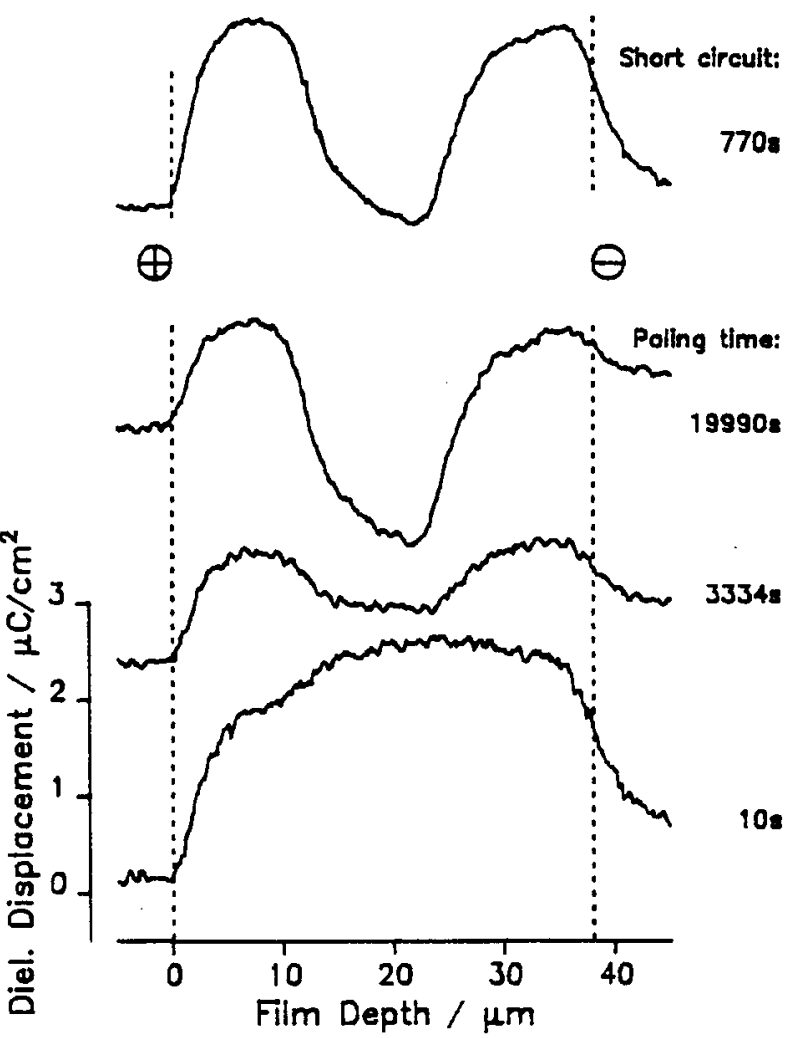

Figure 5: Piezoelectricity profile in a $+/ 0 /+$ PVDF trimorph (top) and its development during application of a relatively weak opposite field to an almost uniformly poled PVDF film (lower part) (after [35]).

The importance of charge injection from the electrodes in the poling process is confirmed by the fact that field application with blocking electrodes does not lead to any permanent polarization in PVDF [36]. The role of the charge trapping at the crystalline-amorphous 
interfaces is, however, not yet fully understood as evidenced by e.g. an anomalous recovery of the polarization in prepolarized PVDF samples heated to 175 or $180^{\circ} \mathrm{C}$ and cooled down to room temperature [37]. Very recently, an additional Maxwell-Wagner interface polarization has been clearly identified in PVDF [38]; its interplay with the ferroelectric polarization in the crystalline phase and the orientation of dipoles in the amorphous phase may eventually help to explain some of the remaining puzzles about piezo- and pyroelectric polymer electrets. Nevertheless, the available poling procedures and polymer materials can now be sufficiently well controlled and reproduced to generate various non-uniform polarization profiles as schematically summarized in Fig. 2 above [29].

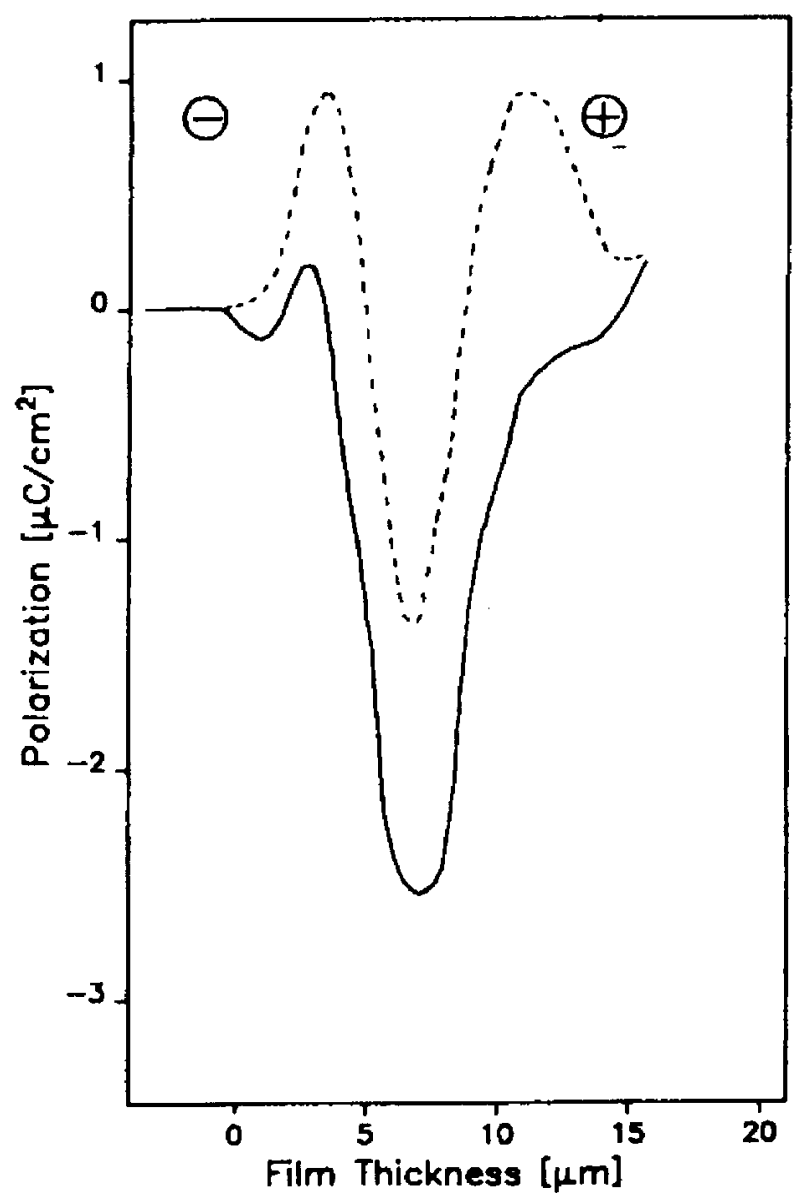

Figure 6: Piezoelectricity profile in a $+/-/+$ PVDF trimorph at the end of (solid line) and after (dashed line) pulsed poling with the polarity indicated (after [33]).

\section{Two-step poling of high- /low $T_{c}$ double layers}

The poling techniques discussed so far are used on single polymer films and thus enable only the preparation of mono- and lowest order multimorphs. Higher-order multimorph preparation is still a challenging task. The use of different polymers permits the preparation of arbitrarily stacked polymer layers, and the heterogeneity of such a stack may be used to advantage for the preparation of true multimorph systems.

G. T. Davis and coworkers at the National Institute of Standards introduced a two-step poling technique for the formation of bimorph structures in layered P(VDFTrFE) copolymer films of different composition [36,39]. The polarization distribution in the bimorph structures was probed by thermal techniques, which rely on the pyroelectric effect of the material. Pyroelectricity in polymers is due to primary and to a large amount secondary pyroelectricity, as a result of the decrease of the dipole number density upon thermal expansion. The pyroelectric coefficient is proportional to the spontaneous polarization and in amorphous polymers to the frozen polarization via $p(x)=\alpha\left(\epsilon_{\infty}+2\right) P(x) / 3$, where $\alpha$ and $\epsilon_{\infty}$ denote the thermal expansion coefficient and the unrelaxed dielectric constant, respectively. Thus depth-resolved monitoring of the dipole orientation is possible by employing thermal techniques.

The layer consisted of $80 / 20$ and $60 / 40$ P(VDFTrFE) copolymers with Curie temperatures above and below $100^{\circ} \mathrm{C}$, respectively. No details were given on the preparation of the bilayer sample. The poling procedure as used by Davis et al. is sketched in Fig. 7: (i) ferroelectric polarization is inscribed into both polymer films in the same direction at room temperature by applying an electric field exceeding the coercive field (Fig. 8(a) left). The thermal-pulse response gives roughly unit steps, as expected for a uniform ferroelectric polarization in both layers (Fig. 8(a) right). (ii) the stack is heated to about $100^{\circ} \mathrm{C}$, which is between the Curietransition temperatures of the two polymer layers. The ferroelectric polarization vanishes in the $60 / 40 \mathrm{P}(\mathrm{VDF}-$ TrFE) copolymer film with the lower Curie temperature. The polarization in the high- $T_{c}$ copolymer, on the other hand, is annealed and stabilized at this temperature. The polarization distribution after annealing (Fig. 8(b) left) was monitored by thermal-pulse probing. When the thermal-pulse is applied to the high- $T_{c}$ side, a step response is observed, followed by a decay 
to half the initial value (Fig. 8(b) right). The different Curie temperatures in the two layers offer the possibility for bimorph preparation. (iii) When a reverse electric field is applied with a field strength lower than the coercive field of the high- $T_{c}$ layer, ferroelectric polarization in the low- $T_{c}$ layer can be induced with reverse orientation by cooling through the lower Curie temperature. The stabilized polarization in the high- $T_{c}$ layer cannot be reversed by the applied electric field. This leads to a structure where the polarization exhibits opposite polarities in the two halves as shown in Fig. 8(c) (left) and experimentally demonstrated by the thermalpulse signals of Fig. 8(c) (right).

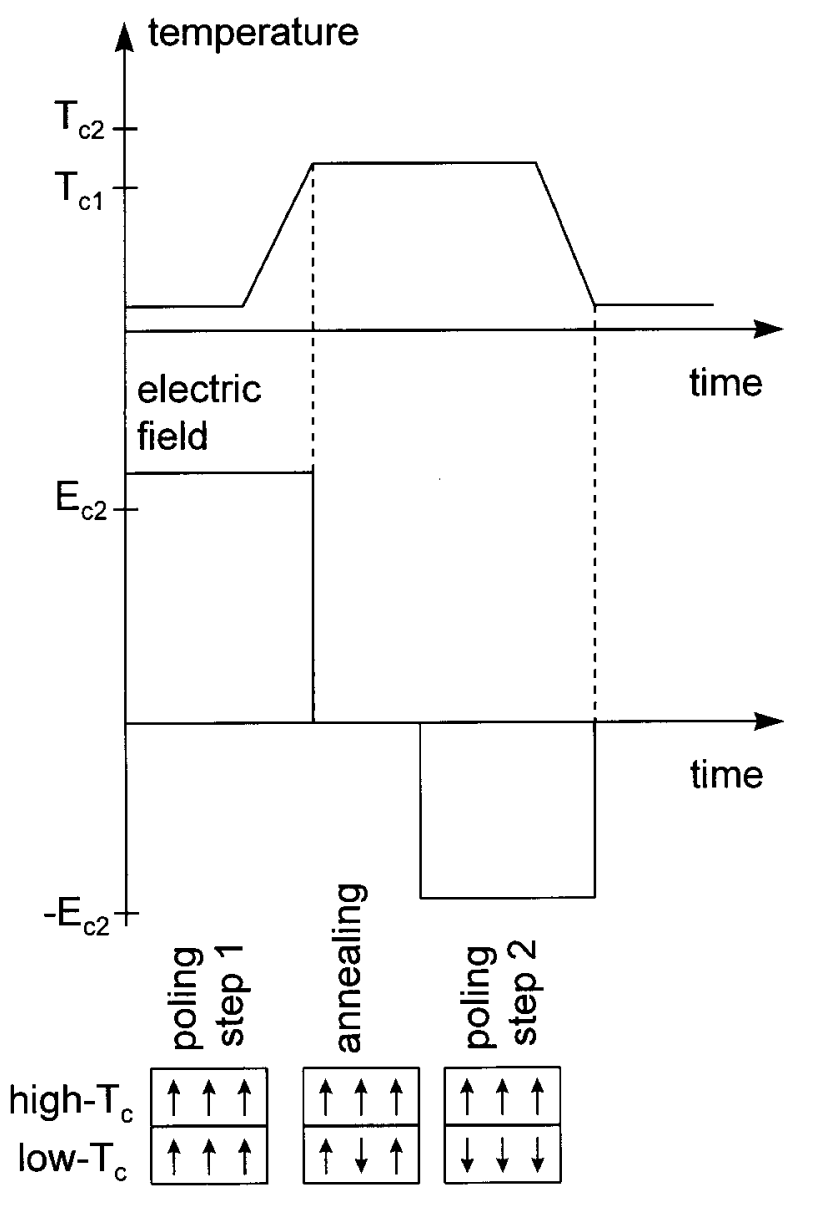

(i) (ii) (iii)

Figure 7: Two-step poling technique for the preparation of multimorphs in ferroelectric polymer multilayers: (i) Inscription of a parallel ferroelectric polarization in both ferroelectric polymer layers, (ii) annealing at a temperature between the two Curie temperatures (polarization in high$T_{c}$ layer stabilized, polarization in low- $T_{c}$ polymer lost), and (iii) reverse-field poling with a field strength smaller than the coercive field in order to avoid switching in the high- $T_{c}$ polymer.
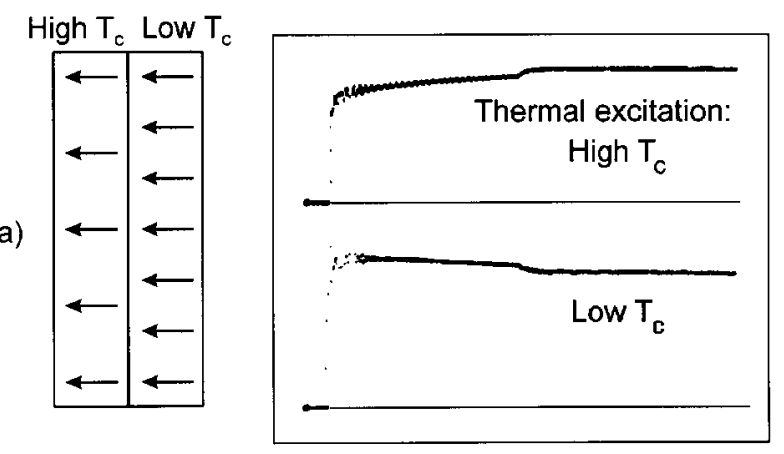

(b)
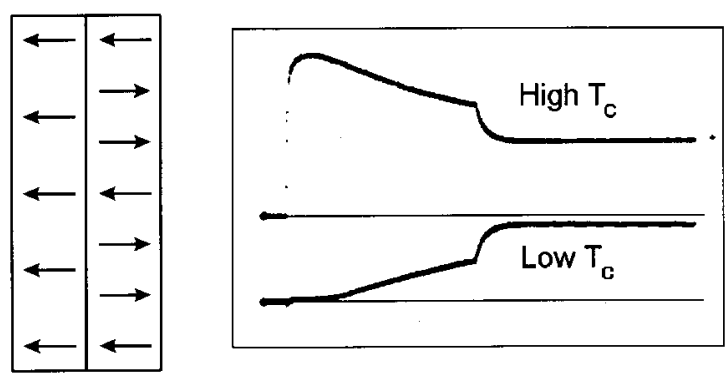

(c)
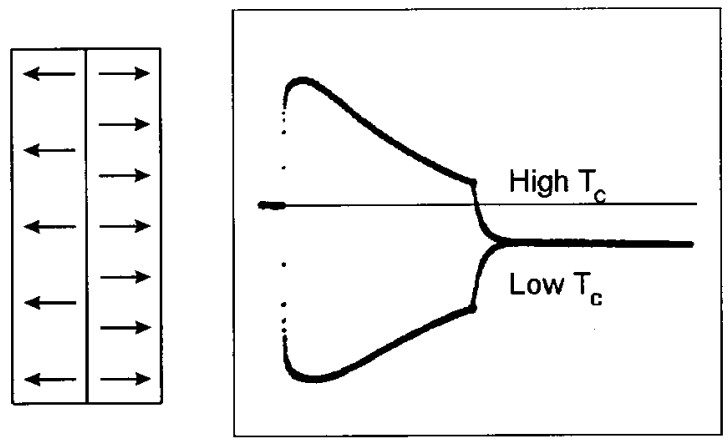

Figure 8: Schematic dipole orientations (left) and thermalpulse responses (right) resulting from the poling steps of Fig. 7: (a) After parallel poling of both layers, (b) after annealing between the Curie temperatures (low- $T_{c}$ polymer depolarized, high- $T_{c}$ polymer with stabilized polarization, and (c) after reverse-field poling (no ferroelectric switching in high- $T_{c}$ polymer, low- $T_{c}$ polymer polarized in opposite direction) (after [36]).

In principle, the technique can be easily adopted for the preparation of higher-order multimorphs, as complex multilayer structures can be produced for example with sophisticated spin-coating procedures. It is important to note that for ferroelectric polymers the first poling step can be performed at room temperature by applying an electric field larger than the highest coercive field. As will be discussed next, the two-step poling has to be modified for amorphous photonics polymers with frozen-in polarization, where a coercive field does not exist. 


\section{Two-step poling of high-/low $T_{g}$ multilayers}

Amorphous, glassy polymers for photonics applications contain chromophores with large dipole moments and hyperpolarizabilities as guest molecules or as side groups. In order to generate the desired nonlinearoptical (NLO) functionality, the chromophores must be oriented within the polymer film in a poling process [40].

In amorphous NLO polymers, the poling-induced dipole orientation is frozen by cooling and keeping the material well below the glass-rubber transition temperature $T_{g}$ after poling in a suitable electric field. Whereas in ferroelectric polymers the ferroelectric phase of the crystallites below the Curie temperature $T_{c}$ is thermodynamically stable, the glassy state in amorphous polymers is thermodynamically metastable, e.g. the dipoles slowly lose their frozen-in orientation [41].

In principle, the preparation of multimorphs based on nonlinear optical polymers is similar to that of bimorphs consisting of ferroelectric polymers, as described above. However, the poling techniques have to be modified in order to account for the different physical mechanisms during the poling processes. Whereas for ferroelectric polymers special attention has to be paid to the strength of the applied electric field during the second poling step in order to avoid switching of the high- $T_{c}$ polymer, with amorphous polymers no such field limitations exist. In this case, the critical parameter is the poling temperature, which has to be carefully chosen in order to achieve the desired structures.

Bauer-Gogonea et al. first proposed a two-step poling procedure for a stack consisting of polymers with different glass-transition temperatures as schematically shown in Fig. 9 [42]. The technique is based on the consecutive thermally-assisted poling at two suitable temperatures above and between the two glass-transition temperatures. For the bilayer, two nonlinear optical polymers with $T_{g}$ 's of $140^{\circ} \mathrm{C}$ and $165^{\circ} \mathrm{C}$, respectively, were used. Details of the poling procedure are found in [42].
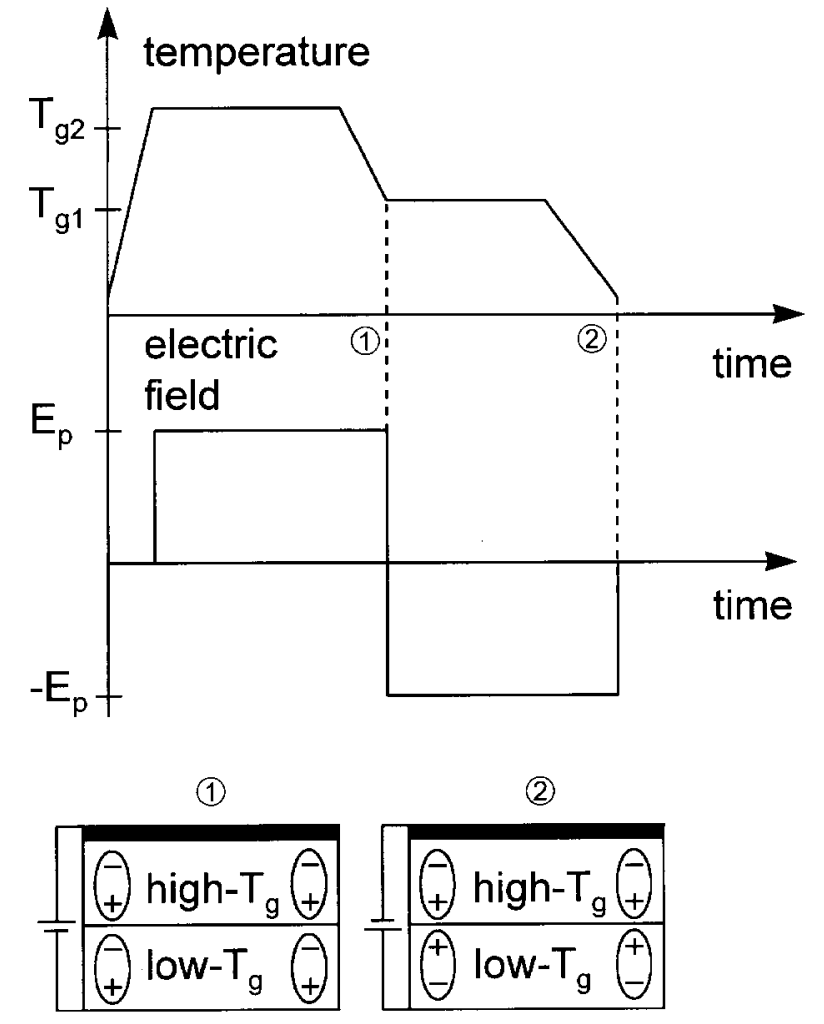

Figure 9: Two-step poling technique for the preparation of multimorphs from amorphous photonics polymers. The technique is based on thermal poling above and between the two glass transitions (after [42]).

Jäger et al. recently demonstrated the preparation of bimorph stacks with NLO-polymer layers; the bimorphs were investigated with thermal-wave measurements during thermally-stimulated depolarization [43]. The thermal excitation was effected with a high modulation frequency of $\mathrm{f}=115 \mathrm{kHz}$, so that the penetration depth of the thermal waves was limited to $0.5 \mu \mathrm{m}$. In this case, as shown schematically in Fig. 10, thermal probing of the polarization is possible in both polymer films. The bimorph was heated at a constant rate while the response was recorded alternately after thermal excitation through the top and bottom metal electrodes, respectively. It is evident from Fig. 10 that the pyroelectric responses of both layers are nearly constant up to the respective glass-transition temperatures and rapidly diminish in the vicinity of the $T_{g}$ 's, where the molecular dipoles become highly mobile. A clear proof for the preparation of a bimorph polarization was thus achieved, as the sign of the pyroelectric coefficient was different in the two polymer films. With a low modulation frequency of $90 \mathrm{~Hz}$, the penetration depth of the thermal-wave exceeds the stack thickness, so that the spatial average of the polarization distribution could 
also be measured (also shown in Fig. 10). In our example, the spatial average of the polarization distribution across the bimorph changed sign during thermally stimulated depolarization (TSD).

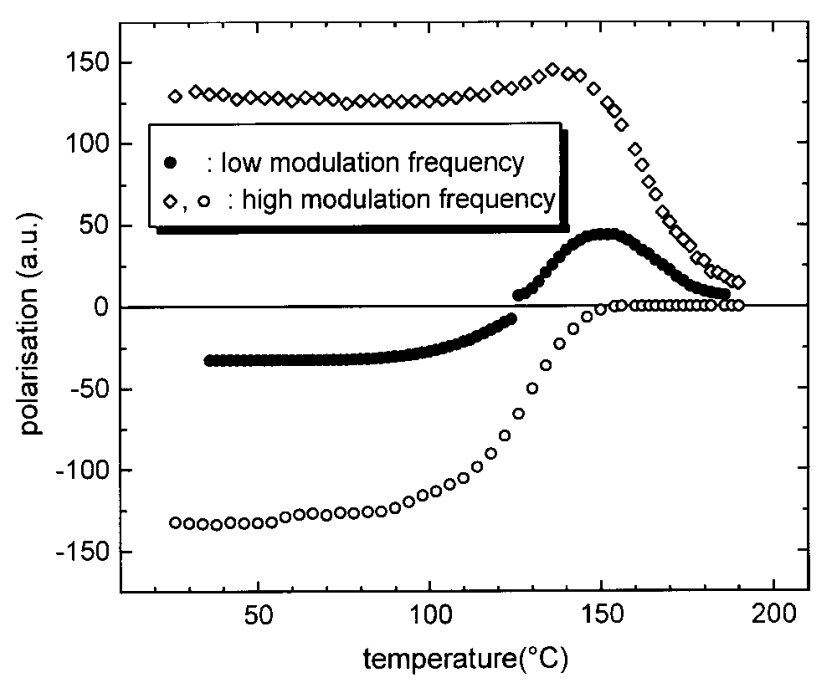

Figure 10: Thermal-wave probing of the polarization in the low- $T_{c}$ and high- $T_{c}$ polymer layers of a bimorph during thermally stimulated depolarization. The sign of the pyroelectric coefficient is different in both layers. With a low modulation frequency (full circles), the spatial average of the dipole polarization can also be probed (after [43]).

Thermal-wave experiments during TSD measurements are intuitive and proof the successful preparation of bimorph structures convincingly. However, for process control, a fast scan of the polarization distribution across the thickness of the polymer films would be more suitable. Thermal wave scans are time consuming and thus less useful for an in-situ process control. Bauer-Gogonea and Bauer [44] recently demonstrated that the thermal-pulse technique can be employed for monitoring the polarization distribution in films with thicknesses on the order of $1 \mu \mathrm{m}$.

Besides electrical techniques, optical techniques have been introduced for the investigation of optically active bi- and multimorph structures. Electro-optical (EO) measurements during poling of polymers allow for the in-situ determination of the orientation polarization and of the mobility of the molecular dipoles in the NLO polymer [43]. Resonantly-enhanced secondharmonic generation has been shown to provide an elegant means for the determination of the polarization distribution in thin NLO polymer films. It could be shown both theoretically and experimentally, that the transmitted SHG from a bimorph layer stems only from the last $0.3 \mu \mathrm{m}$ of the stack, due to the large absorption of the second-harmonic light [44]. The most important advantage of the optical SHG technique is the possible use on waveguide device structures including buffer layers (see below).

It should be mentioned here, that the techniques proposed for NLO polymers may be also interesting for amorphous piezoelectric polymers, such as vinylidenecyanide-based copolymers, which can be processed to have different glass-transition temperatures.

\section{Non-uniform photo-induced poling}

Most chromophore dipoles in NLO polymers can undergo reversible trans-cis-trans isomerization cycles, if illuminated by light of suitable wavelengths within the absorption band of the chromophore (Fig. 11). Dumont and coworkers showed that the trans-cis-trans isomerization cycles strongly enhance the molecular mobility of the chromophore dipoles, thereby allowing for electric-field poling even well below the glass-transition temperature [45].
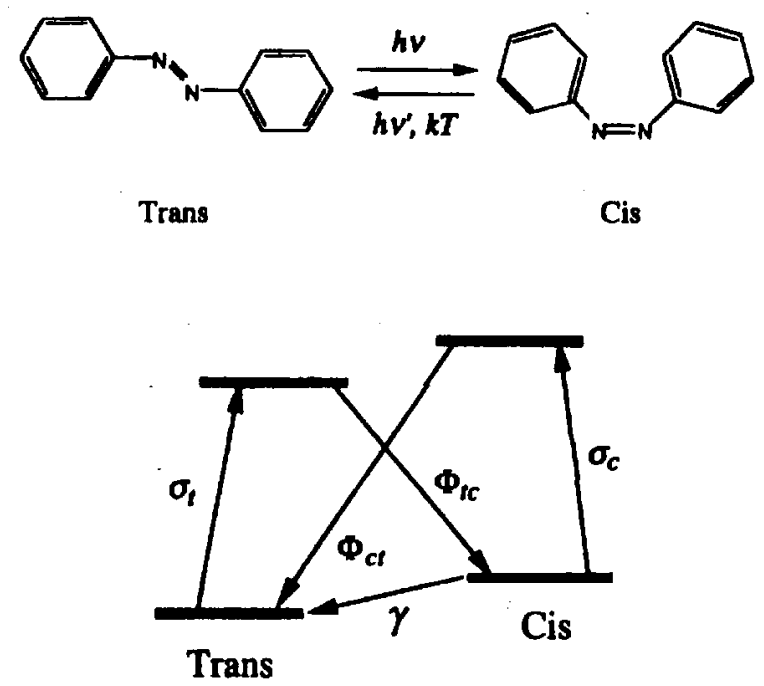

Figure 11: Trans-cis isomerization cycles of azochromophore molecules. The trans-cis transition significantly enhances the mobility of the chromophore dipoles and enables orientation much below the glass-transition temperature (after [45]).

As the pump light used for trans-cis isomerization has a large absorption coefficient on the order of $10^{5} \mathrm{~cm}^{-1}$, the penetration depth of the pump light is limited to thicknesses on the order of $1 \mu \mathrm{m}$. BauerGogonea et al. showed that the dipole orientation during photo-induced poling is limited [46], the poled poly- 
mer film thickness being given by the penetration depth of the pump light. Based on this observation, a twostep poling process, sketched in Fig. 12, was developed which enabled the fabrication of a bimorph stack in a single NLO-polymer layer [42,46]. Successful bimorph preparation was proved by thermal-wave probing of the polarization distribution at room temperature and during thermally-stimulated depolarization. Fig. 13 shows the pyroelectric response and the TSD current during heating of the polymer at a constant rate. Both the pyroelectric response and the TSD current show the behavior expected for a bimorph structure.
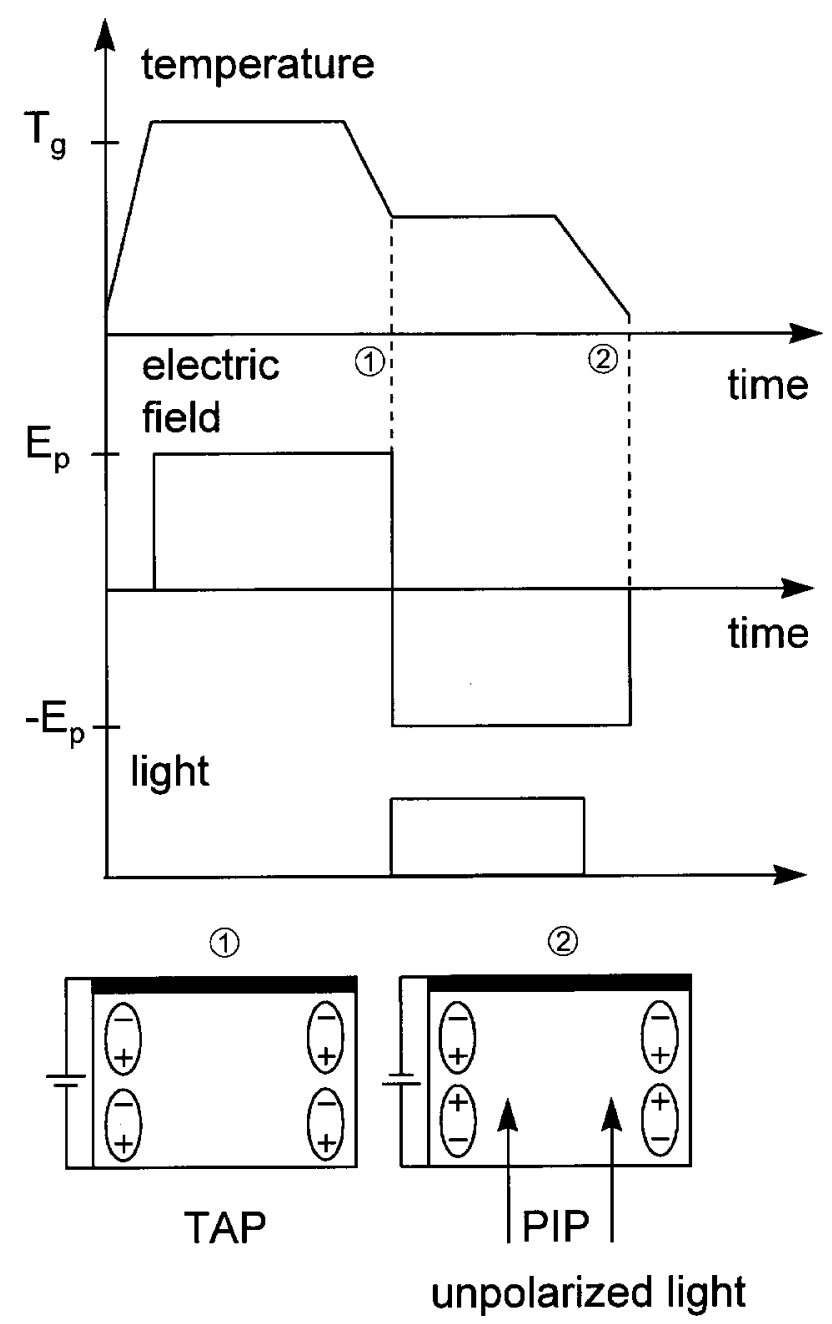

Figure 12: Preparation of bimorphs in a single nonlinear optical polymer film, based on the combination of thermal poling above and photo-induced poling below the glass transition. During the latter step, the dipoles are re-oriented only within the penetration depth of the pump light (after [42]).



Figure 13: Thermal-wave probing of the spatial average of the polarization and corresponding TSD current in a nonlinear optical polymer bimorph poled with the two-step method of Fig. 12 (after [46]).

Khanarian and coworkers at Hoechst Celanese $[47,48]$ introduced another technique for the preparation of NLO polymer-based multimorphs. The NLO polymer is spin-coated onto a silicon substrate, which has been covered with a water-soluble polymer film. After poling the NLO polymer (either electrode or corona poling may be employed), small squares were cut into the films by laser ablation. The wafer was then placed under water until the water soluble polymer between the NLO polymer and the silicon substrate was dissolved, so that the square pieces of NLO polymer floated to the water surface. The pieces were picked up and put between glass slides, so that the poling direction alternated. Stacks with a total number of 52 polymer layers and a thickness of $300 \mu \mathrm{m}$ were demonstrated with this technique. The feasibility of the approach was demonstrated by the observation of quasi-phase matched second-harmonic generation. A multimorph preparation technique was suggested which makes use of conventional polymer processing technology, e.g. film extrusion, hot rolling, stretching and poling and finally cold rolling and laminating to the desired form. Unfortunately, it seems that so far no demonstration of the feasibility of this very interesting proposal has been reported.

\section{Suggested and demon- strated applications}

Multimorph structures may be useful in various applications in diverse fields of physics. A vast amount of information on the use of bi- and multimorphs in piezoelectric applications can be found in the book $[49,50]$ 
and in references quoted therein. In the following, only a few developments, mainly in the field of photonics, are briefly discussed.

A rather exhaustive study of possible piezoelectricpolymer bending-device applications, and of the materials properties required for them, was carried out by Toda around $1980[51,52]$. These devices were all demonstrated with stacks of more or less uniformly poled piezoelectric-polymer films. The use of similarly layered transducers for Barker-coded pulse-echo transduction [2] and for transverse shear-mode operation [53] was also reported recently. The poling methods outlined in this review should enable the manufacture of monolithic devices for these and other applications. Experimental investigations of this possibility include the above-mentioned work on the bending behavior of electron-beam-poled monomorphs [28,29].

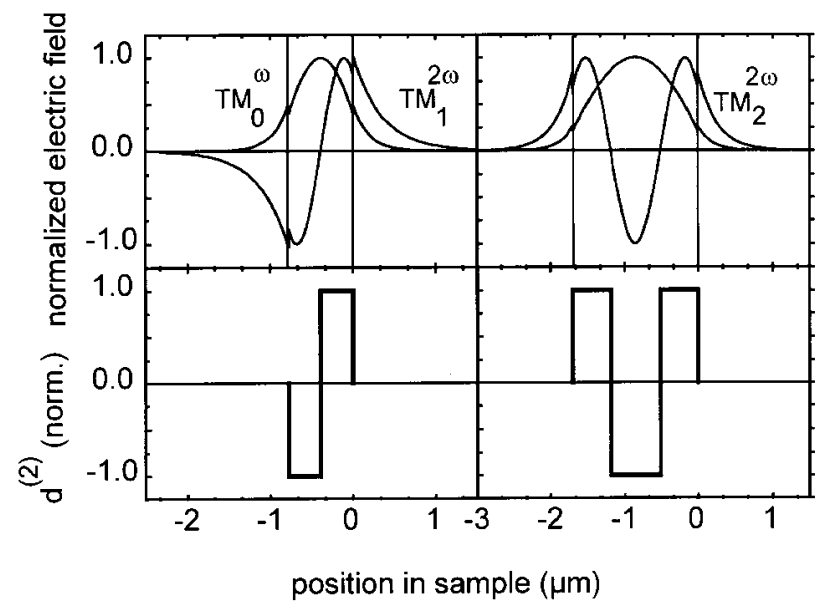

Figure 14: Guided modes in polymer waveguides (top) and corresponding nonlinearity depth profiles as required for phase-matched second-harmonic generation with optimized overlap integrals (after [53]).

Multimorphs are also important in waveguideoptical second-harmonic generation (SHG) with optimized overlap integral [43,54]. Applications of this process are found in optical telecommunication technology. In waveguides light propagates in modes, schematically sketched in the top part of Fig. 14. Optical SHG in waveguides is a mode-conversion process, between the fundamental light mode $\left(T M_{n}^{\omega}, n=0,1,2, \ldots\right)$ at frequency $\omega$, and the $\mathrm{SHG}$ mode $\left(T M_{k}^{\omega}, k=0,1,2, \ldots\right)$ at frequency $2 \omega$. Efficient conversion only occurs, if the modes have the same group velocity (phasematching). Optical dispersion in polymers prevents phase-matched $T M_{0}^{\omega}-T M_{0}^{2 \omega}$ mode-conversion. Efficient mode-conversion is possible solely between the zero-order fundamental $T M_{0}^{\omega}$ and higher-order SHG modes $T M_{k}^{2 \omega}, k>0$. However, it can be shown that the nonlinearity must be multimorph-like across the polymer thickness in this case (Fig. 14 bottom), as possible with the poling techniques discussed above.
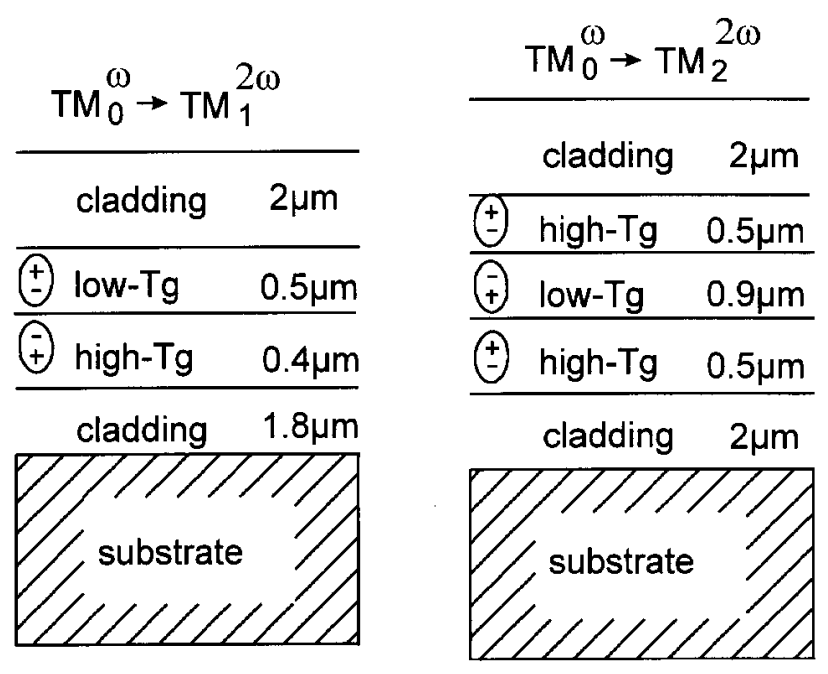

Figure 15: Layer sequences of bi- and trimorph waveguides for phase-matched second- harmonic generation with optimized overlap integrals (after [53]).

Wirges et al. [54] demonstrated efficient waveguide SHG in bi- and trimorph structures, shown schematically in Fig. 15. The waveguides were poled with the two-step poling process for NLO polymers, as introduced above. Large SHG conversion efficiencies, comparable to inorganic ferroelectric $\mathrm{LiNbO}_{3}$ waveguide structures were obtained in first experiments, with a large scope for improvement.

Finally it must be noted that the use of NLO polymer films is not restricted to thin waveguide geometries, for example according to the proposal of Khanarian and coworkers at Hoechst Celanese $[47,48]$ multimorphs can be prepared from bulk polymer films.

\section{Conclusions}

Poling and probing techniques for polymer-electret monomorphs, bimorphs, and multimorphs were reviewed. Typical results were discussed as examples for the exciting possibilities that the combination of in-situ and post-process control offers for the preparation of rather complex depth profiles of the polarization (and thus also of the piezo- and pyroelectric or nonlinear optical effects). A range of suggested and demonstrated applications was briefly introduced. It is to be hoped 
that the required polymer materials as well as the techniques developed during the last three decades will be further refined in the near future so that some of the proposed applications may become reality.

\section{Acknowledgments}

The authors are indebted to Prof. Dr. Gerhard M. Sessler and Dr. Wolfgang Künstler for stimulating discussions and valuable comments on the manuscript and to the Deutsche Forschungsgemeinschaft as well as to the Fonds zur Förderung der wissenschaftlichen Forschung in Österreich for partial financial support.

\section{References}

1 Jacques et Pierre Curie, "Contractions et dilatations produites par des tensions électriques dans les cristaux hémièdres à faces inclinées" (Electric-field induced contractions and expansions in hemihedral crystals with inclined faces), C. R. Acad. Sci. 93, 1137 (1881).

2 Q. Zhang, P. A. Lewin, and Ph. E. Bloomfield, IEEE Trans. Ultrason. Ferroel. Freq. Contr. UFFC-44, 1148 (1997), and references therein.

3 P. Jaeger, Ann. Phys., Ser. 5, 21, 481 (1934).

4 B. Gross, Charge storage in solid dielectrics, Elsevier, Amsterdam 1964, in particular section IV.7., pp. 92-94.

5 B. Gross and R. J. de Moraes, J. Chem. Phys. 37, 710 (1962).

6 R. L. Peterson, G. W. Day, P. M. Gruzensky and R. J. Phelan Jr., J. Appl. Phys. 45, 3296 (1974).

7 R. J. Phelan, Jr., R. L. Peterson, C. A. Hamilton and G. W. Day, Ferroelectrics 7, 375 (1974).

8 R. E. Collins, Appl. Phys. Lett. 26, 675 (1975).

9 R. E. Collins, J. Appl. Phys. 47, 4804 (1976).

10 R. E. Collins, Rev. Sci. Instrum. 48, 83 (1977).

11 P. Laurenceau, J. Bell, G. Dreyfus and J. Lewiner, C. R. Acad. Sci. Paris, Ser. B, 283, 135 (1976).

12 P. Laurenceau, G. Dreyfus and J. Lewiner, Phys. Rev. Lett. 38, 46 (1977).

13 C. Alquié, G. Dreyfus and J. Lewiner, Phys. Rev. Lett. 47, 1483-1487 (1981).

14 A. G. Rozno and V. V. Gromov, Sov. Tech. Phys. Lett. 5, 266 (1979).

15 A. G. Rozno and V. V. Gromov, Russ. J. Phys. Chem. 54, $1482(1980)$.

16 A. S. DeReggi, C. M. Guttman, F. I. Mopsik, G. T. Davis and M. G. Broadhurst, Phys. Rev. Lett. 40, 413 (1978).

17 S. B. Lang and D. K. Das-Gupta, J. Appl. Phys. 59, 2151 (1986).

18 S. Bauer, J. Appl. Phys. 75, 5306 (1994).
19 R. Gerhard-Multhaupt, G. M. Sessler, J. E. West, K. Holdik, M. Haardt, and W. Eisenmenger, J. Appl. Phys. 55, 2769 (1984).

20 C. Alquié and J. Lewiner, Rev. Phys. Appl. 20, 395 (1985).

21 W. Eisenmenger and M. Haardt, Solid-State Commun. 41, 917 (1982).

22 M. A. Marcus, Ferroelectrics 32, 149 (1981).

23 M. A. Marcus, J. Appl. Phys. 52, 6273 (1981).

24 G. T. Davis, "Piezoelectric and pyroelectric polymers", in C. P. Wong (Ed.), Polymers for electronic and photonic applications, Academic Press, San Diego 1992, pp. 435-465, in particular Fig. 6, p. 447.

25 B. Gross, G. M. Sessler and J. E. West, J. Appl. Phys. 45, 2841 (1974).

26 R. Gerhard-Multhaupt, W. Künstler, G. Eberle, W. Eisenmenger and G. Yang, "High space-charge densities in the bulk of fluoropolymer electrets detected with piezoelectrically generated pressure steps", in J. C. Fothergill and L. A. Dissado (Eds.), Space charge in solid dielectrics, The Dielectrics Society, Leicester 1998, pp. 123-132, and references therein.

27 B. Gross, R. Gerhard-Multhaupt, A. Berraissoul and G. M. Sessler, J. Appl. Phys. 62, 1429 (1987).

28 E. Fukada, G. M. Sessler, J. E. West, A. Berraissoul and P. Günther, J. Appl. Phys. 62, 3643 (1987).

29 G. M. Sessler and A. Berraissoul, Ferroelectrics 76, 489 (1987).

30 G. M. Sessler and A. Berraissoul, IEEE Trans. Electr. Insul. EI-24, 249 (1989).

31 D. Schilling, K. Dransfeld, E. Bihler, K. Holdik, and W. Eisenmenger, J. Appl. Phys. 65, 269 (1989).

32 G.-M. Yang, S. Bauer-Gogonea, G. M. Sessler, S. Bauer, W. Ren, W. Wirges and R. Gerhard-Multhaupt, Appl. Phys. Lett. 64, 22 (1994).

33 M. Womes, E. Bihler and W. Eisenmenger, IEEE Trans. Electr. Insul. EI-24, 461 (1989).

34 E. Bihler, K. Holdik and W. Eisenmenger, IEEE Trans. Electr. Insul. EI-24, 541 (1989).

35 G. Eberle, B. Dehlen, and W. Eisenmenger, "Time development of multiple polarization zones in PVDF", 1993 IEEE Ultrason. Symp. Proc., 1, pp. 529 (1993).

36 G. M. Sessler, D. K. Das-Gupta, A. S. DeReggi, W. Eisenmenger, T. Furukawa, J. A. Giacometti and R. Gerhard-Multhaupt, IEEE Trans. Electr. Insul. EI-27, 872 (1992), in particular pp. 875-884.

37 G. Eberle and W. Eisenmenger, IEEE Trans. Electr. Insul. EI-27, 768 (1992).

38 D. Rollik, S. Bauer and R. Gerhard-Multhaupt, J. Appl. Phys. 85, to be published in March 1999.

39 G. T. Davis, A. S. DeReggi, F. I. Mopsik and S. C. Roth, "Polarization distribution in multilayer films of vinylidene fluoride trifluoroethylene copolymers", presented at 4th USA- Japan Workshop on Dielectric and Piezoelectric Materials, National Institute of Standards and Technology (Gaithersburg, MD), 30 Oct.-2. Nov. 1988. 
40 for a recent survey see: S. Bauer-Gogonea and R. Gerhard-Multhaupt, IEEE Trans. Diel. Electr. Insul. 3, 677 (1996).

41 K. D. Singer, J. E. Sohn and S. J. Lalama, Appl. Phys. Lett. 49, 248 (1986).

42 S. Bauer-Gogonea, S. Bauer, W. Wirges and R. Gerhard-Multhaupt, Ann. Physik 4, 355 (1995).

43 M. Jäger, G. I. Stegeman, S. Yilmaz, W. Brinker, W. Wirges, S. Bauer-Gogonea, S. Bauer, M. Ahlheim, M. Stähelin, B. Zysset, F. Lehr, M. Diemeer and M. C. Flipse, J. Opt. Soc. Am. B, 15, 781 (1998).

44 S. Bauer-Gogonea and S. Bauer, "In-situ profiling of dipole polarization distributions in poled nonlinear optical polymers with electrothermal and optical techniques", Chem. Phys., submitted.

45 Z. Sekkat and M. Dumont, Appl. Phys. B 54, 486 (1992).

46 S. Bauer-Gogonea, S. Bauer, W. Wirges and R. Gerhard-Multhaupt, J. Appl. Phys. 76, 2627 1994).

47 G. Khanarian, M. A. Mortazavi and A. J. East, Appl. Phys. Lett. 63, 1462 (1993).
48 M. A. Mortazavi and G. Khanarian, Opt. Lett. 19 $1290(1994)$.

49 T. T. Wang, J. M. Herbert and A. M. Glass (eds.), The applications of ferroelectric polymers, Blackie \& Son Ltd., (Glasgow and London 1988).

50 H. S. Nalwa (ed.), Ferroelectric polymers, Marcel Dekker, (1995).

51 M. Toda, J. Appl. Phys. 51, 4673 (1980), and references therein.

52 M. Toda, Ferroelectrics 32, 127 (1981), and references therein.

53 K. Omote and H. Ohigashi, IEEE Trans. Ultrason. Ferroel. Freq. Contr. UFFC-43, 312 (1996).

54 W. Wirges, S. Yilmaz, W. Brinker, S. Bauer-Gogonea, S. Bauer, M. Jäger, G. I. Stegeman, M. Ahlheim, M. Stähelin, B. Zysset, F. Lehr, M. Diemeer and M. C. Flipse, Appl. Phys. Lett. 70, 3347 (1998). 